\title{
Performance Comparison of IEEE 802.11p and IEEE 802.11b for Vehicle-to-Vehicle Communications in Highway, Rural, and Urban Areas
}

\author{
B. E. Bilgin ${ }^{1}$ and V. C. Gungor ${ }^{1,2}$ \\ ${ }^{1}$ Computer Engineering Department, Bahcesehir University, 34353 Istanbul, Turkey \\ ${ }^{2}$ Department of Computer Engineering, Abdullah Gul University, 38039 Kayseri, Turkey \\ Correspondence should be addressed to B. E. Bilgin; bilalerman.bilgin@bahcesehir.edu.tr
}

Received 24 June 2013; Accepted 13 September 2013

Academic Editor: Aboelmagd Noureldin

Copyright (C) 2013 B. E. Bilgin and V. C. Gungor. This is an open access article distributed under the Creative Commons Attribution License, which permits unrestricted use, distribution, and reproduction in any medium, provided the original work is properly cited.

Communication between vehicles has recently been a popular research topic. Generally, the Vehicle-to-Vehicle (V2V), Vehicle-toInfrastructure (V2I), and Infrastructure-to-Infrastructure (I2I) communications applications can be divided into two sections: (i) safety applications and (ii) nonsafety applications. In this study, we have investigated the performance of IEEE 802.11p and IEEE $802.11 \mathrm{~b}$ based on real-world measurements and radio propagation models of $\mathrm{V} 2 \mathrm{~V}$ networks in different environments, including highway, rural, and urban areas. Furthermore, we have investigated the most used V2V mobility models and simulation tools. Comparative performance evaluations show that the IEEE 802.11p achieves higher network throughput, low end-to-end delay, and higher delivery ratio compared to IEEE 802.11b. Overall, our main objective is to describe potential advantages, research challenges, and applications of V2V networks and show how IEEE 802.11p and IEEE 802.11b will perform under different radio propagation environments.

\section{Introduction}

Vehicle-to-Vehicle (V2V) communication has recently become a hot topic in both academy and automotive industries [1-3]. The communication between vehicles helps to improve road safety. In these networks, vehicles act like sensors and transmit the warning messages to other vehicles in communication range or receive the messages from other vehicles. Drivers can easily detect any abnormal or potentially dangerous situations, such as traffic accidents and traffic jam, by receiving telematics information, including location and speed information.

The main applications of $\mathrm{V} 2 \mathrm{~V}$ technology include road safety applications, including accident warning, and blind spot warning, lane change warning, intersection warning, emergency vehicle warning [1-6]. Also there are efficiency and commercial applications, including route guidance systems, transportation congestion systems, tolling payment, and fleet control.
The key factor in V2V communication networks is the mobility of nodes. In addition to mobility challenges, in these networks vehicles should also obey the traffic specific rules and follow the available roads. Therefore, a special form of an ad hoc network for V2V systems has emerged, which has been called vehicular ad hoc network (VANET). Some of the issues that affect the performance of VANETs are high mobility, signal fading, packet collisions, radio interferences on transmitting data, and so forth.

The illustration of $\mathrm{V} 2 \mathrm{~V}$ communication is shown in Figure 1. In general, there are three types of V2V communications [5]. The first one is communication between vehicles $(\mathrm{V} 2 \mathrm{~V})$, in which vehicles can share information about road, can transmit accident or collision information, and so forth. The second one is communication between vehicles and infrastructures (V2I), in which, road side units (RSU) can transmit commercial advertisements, vehicles can pay toll or parking payments, and so forth. The third one is communication between infrastructures (I2I), in which the 


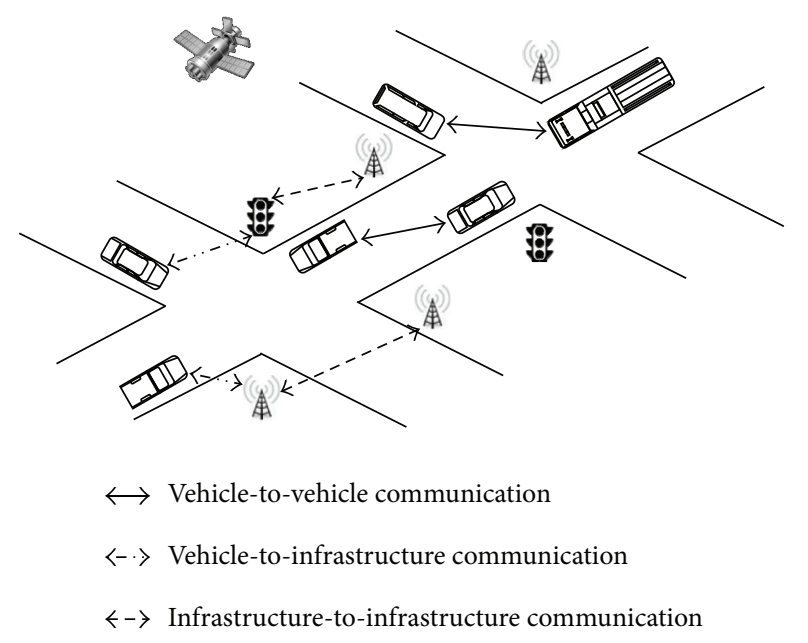

Figure 1: Vehicle-to-Vehicle (V2V), Vehicle-to-Infrastructure (V2I), and Infrastructure-to-Infrastructure I2I networks.

RSUs can exchange important data that is received from the vehicles with each other.

The communication standards in vehicular communication are dedicated short range communication (DSRC) and IEEE 1609 [3, 5]. DSRC is used for short range communication in V2V and V2R applications. The features of DSRC are low communication delay and high data transfers. DSRC has 7 channels, and they have been allocated in this order: first channel is used for safety communications, second channel is used for critical safety communications, third channel is used for high power public safety communications, and the rest of the channels are used for either safety or nonsafety communications. The standards of DSRC are different in Japan, Europe, and USA. In each of the three regions DSRC has different features. For example, communication range is 30 meters in Japan, 15-20 meters in Europe, and 1000 meters ( $\max$ ) in USA. The data rate in Japan is $1-4 \mathrm{Mbps}, 250 \mathrm{Kbps}$ in Europe, and 3-27 Mbps in USA. The comparison of regional differences in DSRC has been summarized in Table 1.

IEEE $802.11 \mathrm{~b}$ protocol may be used for wireless communication in V2V. However, some challenges such as vehicle speed, traffic patterns, and high mobility, affect the communication, that is, establishing communication between vehicles that approach from opposite direction. To address these challenges, IEEE 1609 standards for wireless access in vehicular environments (WAVE) have emerged. IEEE 1609 has resource manager protocols, security services protocols, networking protocols, and multichannel operation protocols. IEEE 802.11p is the updated version of IEEE 802.11b standard that works on data link and physical layers and enables communication between high speed vehicles. WAVE protocol works on the rest of the OSI layers. The physical layer values of the IEEE 802.11b and IEEE 802.11p have been summarized in Table 2.

Since real-time field tests are very expensive and topology of network is difficult to construct due to requirements of high number of vehicles, detailed performance evaluations are very important to develop protocols for V2V applications.
In this paper, we have compared the IEEE 802.11b and IEEE 802.11p protocols in different environments, including rural, urban, and suburban areas using MObility model generator for VEhicular networks (MOVE) simulator tool. We have evaluated the performance of IEEE 802.11p and IEEE $802.11 \mathrm{~b}$ in terms of average delay, delivery ratio, and network throughput. In this work, we also introduce simulators, and mobility models that are widely used in VANET simulations. Here, our objective is to describe potential advantages and applications of V2V networks and show how IEEE 802.11p and IEEE $802.11 \mathrm{~b}$ will perform under different propagation environments.

The remainder of this paper is organized as follows. In Section 2, an overview of the related work on V2V communications is presented. In Section 3, V2V applications and requirements are summarized, and research challenges are presented. In Section 4, V2V simulators and mobility models are summarized. In Section 5, comparative performance results are shown. Finally, the paper is concluded in Section 6.

\section{Related Work}

In the related literature, there are some studies about measuring propagation channels for V2V communications [4, 9-11]. Reliable V2V communications require accurate propagation channel models. This section provides an overview of $\mathrm{V} 2 \mathrm{~V}$ propagation channel measurements. The propagation of $\mathrm{V} 2 \mathrm{~V}$ is not the same as cellular channels. The time and frequency selectivity in V2V are different compared to cellular communication [1].

In [9], the authors have made some empirical measurements for $\mathrm{V} 2 \mathrm{~V}$ communications. They have used prototype of IEEE 802.11p equipment. Two vehicles are equipped with CVIS OBU with a microwave communication module (MCM). Their experiment environments are highway, rural, and urban areas. They consider different speeds and distances in their measurements.

In [12], the authors have measured the channel by using log-normal shadowing path loss model for highway, rural, and urban environments at $5.9 \mathrm{GHz}$. Different vehicle movement simulation scenarios such as driving opposite direction and driving in same direction have been considered. They also have considered LOS and NLOS conditions. After the measurements, they give the path loss and shadowing deviation parameters for three environments.

In [13], the authors have made channel measurements in highway and rural driving environments by using onroad vehicular test bed equipment. They have also used programmable laboratory instruments in their measurements. Their studies include the narrow-band measurements, Doppler-delay measurements, antenna pattern's effects, and variations of passing vehicles. Frequency spectrum of signals has been used to compute the received signal strength. Based on the channel measurements, they have reported dual-slope log-normal shadowing path loss parameters.

In [14], the authors have measured the V2V propagation channel in realistic suburban environments in Pittsburgh, Pennsylvania, at $5.9 \mathrm{GHz}$. To enable the dynamic measurements, they include differential global positioning system 
TABLE 1: Regional differences in DSRC.

\begin{tabular}{lccc}
\hline Features & Japan & Europe & USA \\
\hline Radio band & $80 \mathrm{MHz}$ & $20 \mathrm{MHz}$ & $75 \mathrm{MHz}$ \\
Data rate & $1-4 \mathrm{Mbps}$ & $250 \mathrm{Kbps}$ & $3-27 \mathrm{Mbps}$ \\
Communication range & $30 \mathrm{~m}$ & $15-20 \mathrm{~m}$ & $1000 \mathrm{~m}(\mathrm{max})$ \\
Radio frequency & $5.8 \mathrm{GHz}$ & $5.8 \mathrm{GHz}$ & $5.9 \mathrm{GHz}$ \\
\hline
\end{tabular}

TABLE 2: PHY layer values of IEEE 802.11b and IEEE 802.11p [7].

\begin{tabular}{lcc}
\hline Parameters & IEEE $802.11 \mathrm{~b}$ & IEEE $802.11 \mathrm{p}$ \\
\hline Channel bandwidth & $20 \mathrm{MHz}$ & $10 \mathrm{MHz}$ \\
Data rates & 1 to $11 \mathrm{Mbps}$ & 3 to $27 \mathrm{Mbps}$ \\
Slot time & $20 \mu \mathrm{s}$ & $16 \mu \mathrm{s}$ \\
SIFS time & $10 \mu \mathrm{s}$ & $32 \mu \mathrm{s}$ \\
Preamble length & $96 \mu \mathrm{s}$ (short), $192 \mu \mathrm{s}$ (long) & $32 \mu \mathrm{s}$ \\
Air propagation time & $<2 \mu \mathrm{s}$ & $<4 \mu \mathrm{s}$ \\
CWmin & 31 & 15 \\
CWmax & 1023 & 1023 \\
\hline
\end{tabular}

(DGPS). To allow dynamic measurements when the vehicles are on way, they include GPS receivers into their measurements. They have used log-normal shadowing path loss model to obtain large scale path loss models. They introduce both empirical data for $\mathrm{V} 2 \mathrm{~V}$ wireless channel modeling and encourage the research community for theoretical modeling studies in V2V.

Although all these studies are valuable for VANET, there is no comprehensive study that compares performance of IEEE 802.11b and IEEE 802.11p in different environments, including rural, urban, and suburban areas in terms of average delay, delivery ratio, and network throughput. Overall, our main objective is to describe potential advantages, research challenges, and applications of V2V networks and show how IEEE 802.11p and IEEE 802.11b will perform under different radio propagation environments.

\section{V2V Applications and Challenges}

3.1. V2V Applications. This section introduces the available and potential applications of $\mathrm{V} 2 \mathrm{~V}$. Basically, there are two types of $\mathrm{V} 2 \mathrm{~V}$ applications, that is, road safety applications and traffic efficiency applications. An overview of $\mathrm{V} 2 \mathrm{~V}$ applications can be summarized as follows.

3.1.1. Road Safety Applications. The objectives of the road safety applications are to decrease the number of traffic accidents and to help the drivers increase their safety. These applications provide information to drivers about collisions that have occurred on that way [8]. Furthermore these applications warn driver when another driver makes immediate break or tries to decrease traffic accident at the road intersections to increase the safety of drivers.

3.1.2. Traffic Efficiecny Applications. The aims of the traffic efficiency applications are improving the traffic flow and coordination of traffic by providing instant traffic information between vehicles and RSUs. These applications try to balance the speed of vehicle and inform the drivers when they try to reach the destination.

The V2V applications have been summarized in Table 3.

\subsection{V2V Challenges}

3.2.1. Routing Challenges. Determining the possible routes in VANETs is hard due to mobility of nodes. The direction, position, and speed of vehicles always change. In this case, even though the source and destination nodes are stable, the location of intermediate nodes changes, which can create packet losses along the path.

3.2.2. Doppler Effect. When two vehicles approach each other due to the Doppler effect, the frequency may be different on the receiver and transmitter side. Therefore, frequency should be regulated on the receiving side.

3.2.3. Hidden Terminal Problem. When there is no centralized communication coordination, the hidden terminal problem occurs in VANETs. This causes collisions when two nodes that are not in same communication range try to transmit data to the same node.

3.2.4. Data Security. The privacy of data may be important in some applications. To increase the security, some encoding mechanisms may be used. However, this causes extra overhead on the data and may affect the system performance.

3.2.5. Delay Constraints. In emergency situation, the warning messages have to be forwarded immediately. Due to mobility of nodes and changing in network topology, latency may increase.

\section{V2V Simulators and Mobility Models}

Since real-time field tests are very expensive and topology of network is difficult to construct due to requirements of 
TABLE 3: V2V applications and requirements [8].

\begin{tabular}{|c|c|c|c|c|}
\hline Application name & Description & Application type & Communication mode & $\begin{array}{l}\text { Minimum } \\
\text { transmission } \\
\text { frequency }\end{array}$ \\
\hline $\begin{array}{l}\text { Emergency Brake Light } \\
\text { Warning }\end{array}$ & $\begin{array}{l}\text { Informs vehicles when } \\
\text { driver brakes }\end{array}$ & Safety application & $\begin{array}{l}\text { Periodic permanent } \\
\text { message broadcasting }\end{array}$ & $10 \mathrm{~Hz}$ \\
\hline Forward Collision Warning & $\begin{array}{l}\text { Detects moving vehicles } \\
\text { directly ahead }\end{array}$ & Safety application & $\begin{array}{l}\text { Cooperation } \\
\text { awareness between } \\
\text { vehicles associated with } \\
\text { unicast }\end{array}$ & $10 \mathrm{~Hz}$ \\
\hline Intersection Movement & $\begin{array}{l}\text { Warns the drivers when } \\
\text { they approach intersections }\end{array}$ & Safety application & $\begin{array}{l}\text { Periodic } \\
\text { message broadcasting }\end{array}$ & $10 \mathrm{~Hz}$ \\
\hline $\begin{array}{l}\text { Blind Spot \& Lane } \\
\text { Changing Assistance }\end{array}$ & $\begin{array}{l}\text { Decreases lateral collisions } \\
\text { by disabling the lane } \\
\text { changing }\end{array}$ & Safety application & $\begin{array}{l}\text { Periodic } \\
\text { message broadcasting }\end{array}$ & $10 \mathrm{~Hz}$ \\
\hline $\begin{array}{l}\text { Do Not Pass (Overtaking) } \\
\text { Warning }\end{array}$ & $\begin{array}{l}\text { Warns the driver not to } \\
\text { overtake or disable lane } \\
\text { changing }\end{array}$ & Safety application & $\begin{array}{l}\text { Broadcasting when } \\
\text { overtaking }\end{array}$ & $10 \mathrm{~Hz}$ \\
\hline $\begin{array}{l}\text { Head On \& Rear End } \\
\text { Collision Warning }\end{array}$ & $\begin{array}{l}\text { Warns the drivers that are } \\
\text { coming from opposite lane } \\
\text { when a traffic accident } \\
\text { occurs }\end{array}$ & Safety application & $\begin{array}{l}\text { Broadcasting when } \\
\text { collision occurred }\end{array}$ & $10 \mathrm{~Hz}$ \\
\hline Collision Risk Warning & $\begin{array}{l}\text { Detects vehicles that have } \\
\text { risk of collision }\end{array}$ & Safety application & $\begin{array}{l}\text { Broadcasting when a risk } \\
\text { occurred }\end{array}$ & $10 \mathrm{~Hz}$ \\
\hline $\begin{array}{l}\text { Hazardous Location } \\
\text { Notification }\end{array}$ & $\begin{array}{l}\text { Informs the drivers when } \\
\text { some obstacles are } \\
\text { discovered on the road }\end{array}$ & Safety application & $\begin{array}{l}\text { Broadcasting when road is } \\
\text { slippery }\end{array}$ & $10 \mathrm{~Hz}$ \\
\hline $\begin{array}{l}\text { Emergency Vehicle } \\
\text { Warning }\end{array}$ & $\begin{array}{l}\text { Broadcasts to all vehicles } \\
\text { and RSUs to open the lane } \\
\text { when an emergency vehicle } \\
\text { is detected }\end{array}$ & Safety application & $\begin{array}{l}\text { Broadcasting when an } \\
\text { emergency vehicle is } \\
\text { detected }\end{array}$ & $10 \mathrm{~Hz}$ \\
\hline Speed Management & $\begin{array}{l}\text { Balances the speed of } \\
\text { vehicle }\end{array}$ & $\begin{array}{l}\text { Traffic efficiency } \\
\text { application }\end{array}$ & $\begin{array}{l}\text { Cooperation } \\
\text { awareness }\end{array}$ & $2 \mathrm{~Hz}$ \\
\hline $\begin{array}{l}\text { Route Guidance \& } \\
\text { Transportation Congestion } \\
\text { Systems }\end{array}$ & $\begin{array}{l}\text { Informs the drivers when } \\
\text { they try to reach the } \\
\text { destination }\end{array}$ & $\begin{array}{l}\text { Traffic efficiency } \\
\text { application }\end{array}$ & $\begin{array}{l}\text { Cooperation } \\
\text { awareness }\end{array}$ & $2 \mathrm{~Hz}$ \\
\hline Fleet Control & $\begin{array}{l}\text { Informs the fleet companies } \\
\text { about their vehicles }\end{array}$ & $\begin{array}{l}\text { Traffic efficiency } \\
\text { application }\end{array}$ & $\begin{array}{l}\text { Cooperation } \\
\text { awareness }\end{array}$ & $2 \mathrm{~Hz}$ \\
\hline
\end{tabular}

high number of vehicles, detailed performance evaluations through simulations are very crucial to develop protocols for $\mathrm{V} 2 \mathrm{~V}$ applications [9]. In the following two subsections, we introduce the widely used VANET simulator tools and mobility models that are used in simulations.

4.1. VANET Simulators. This subsection introduces the simulators for VANET study. There are two types of simulator for VANET.

(i) Network simulators that are related to network applications and protocols studies: in these simulators, trace files that have been generated by traffic simulators are evaluated.

(ii) Traffic simulators that are related to traffic and transportation studies: in these simulators, initial positions, speeds, and type of vehicles are defined.

4.1.1. MOVE. The MObility model generator for VEhicular networks (MOVE) is a Java based VANET simulator and runs commands of Simulation of Urban MObility (SUMO) at background with GUI support [15]. In this simulator, maps can be defined in three different ways. Researchers can manually define the map, maps can be generated automatically by researchers, and real maps from topologically integrated geographic encoding and referencing (TIGER) and Google Earth can be imported. The movement of vehicles can also be created manually or automatically. MOVE also generates trace files for network simulators, including NS-2 and QualNet to simulate the movements of vehicles.

4.1.2. TRANS. Traffic and network simulator (TRANS) is Java based VANET simulator with GUI support. It combines NS-2 and SUMO [16]. SUMO generates trace files and parses them to NS-2 simulator. TraNs has a light version called TraNs Lite that just generates mobility models without generating trace files for NS-2.

4.1.3. SUMO. SUMO is C++ based microscopic and open source traffic simulator with GUI support [17]. It can generate 
TABLE 4: Simulators Comparison.

\begin{tabular}{|c|c|c|c|c|c|c|}
\hline $\begin{array}{l}\text { Simulator } \\
\text { name }\end{array}$ & Language & $\begin{array}{c}\text { Network } \\
\text { simulator support }\end{array}$ & $\begin{array}{c}\text { GUI } \\
\text { support }\end{array}$ & $\begin{array}{c}\text { Map } \\
\text { support }\end{array}$ & $\begin{array}{c}\text { Movement Of } \\
\text { vehicles }\end{array}$ & $\begin{array}{l}\text { Simulation } \\
\text { scale }\end{array}$ \\
\hline MOVE & Java & NS-2, QualNet & Yes & $\begin{array}{l}\text { TIGER, Google } \\
\text { Earth }\end{array}$ & $\begin{array}{c}\text { Manually, } \\
\text { automatically }\end{array}$ & No information \\
\hline TRANS & Java & NS-2 & Yes & $\begin{array}{c}\text { TIGER, Shapefile } \\
\text { Maps }\end{array}$ & No information & Up to 3000 vehicles \\
\hline SUMO & $\mathrm{C}++$ & $\begin{array}{l}\text { NS-2, QualNet, } \\
\text { Ansim }\end{array}$ & Yes & $\begin{array}{c}\text { OpenStreetMap, } \\
\text { TIGER }\end{array}$ & Manually & $\begin{array}{l}\text { Up to } 100000 \text { vehicles } \\
\text { and } 10000 \text { edges }\end{array}$ \\
\hline VanetMobiSim & Java & $\begin{array}{l}\text { NS-2, Glosim, } \\
\text { QualNet }\end{array}$ & Yes & TIGER & No information & No information \\
\hline
\end{tabular}

mobility trace files to NS-2, QualNet, and Ansim. Network maps can be generated manually, or real-world maps from OpenStreetMap and TIGER. It is also compatible with network maps that are generated by other traffic simulators such as MATsim, OSM, RoboCup, Shapefiles, VISUM, and Vissim. SUMO also supports different vehicle types and way rules. 100,000 vehicles and 10,000 edges can be managed in simulations. Each vehicle has its own route in simulations.

4.1.4. VanetMobiSim. Vanet mobility simulation (VanetMobiSim) is Java based micromobility and macromobility VANET simulator with GUI support [4]. It is the extension version of CanuMobiSim. Networks maps can be generated manually and randomly. It can generate trace files for network simulators, including NS-2, Glosim, and Qualnet. VanetMobiSim also considers road topology, road structure, and traffic signs.

Table 4 summarizes the comparison of the most used V2V simulators in terms of programming language, network simulator support, GUI support, map support, movement of vehicles, and simulation scale.

4.2. Mobility Models. This subsection introduces the mobility models that are used in VANET simulations. Each model uses different parameters to create the path of vehicles. In mobility models concept, there are two important levels.

(i) Macroscopic mobility refers to streets, traffic lights, speed limits, number of lanes, building, traffic signs, and so forth.

(ii) Microscopic mobility refers to vehicle movements and behavior of drivers according to their age, mood, and sex.

There are some factors that significantly affect vehicles' mobility pattern. Some of these factors are as follow:

(i) vehicle speed,

(ii) streets layout,

(iii) traffic signs (stop signs, lights).

4.2.1. Freeway Model. In this model, movement pattern of mobile nodes in freeways is simulated [18]. There is no intersection in this model. In other words, there are vertical or horizontal lanes on the map. Nodes are randomly distributed and use this speed formula:

$$
V(t+1)=V(t)+\operatorname{rand}(-1,1) * a(t),
$$

where $V(t)$ represents the speed of vehicle at time $t, a(t)$ represents the vehicle's acceleration at time $t$, and rand $(-1,1)$ means a random number between -1 and 1 . Nodes cannot change their lane and a node cannot pass another node that is ahead of itself in this model.

4.2.2. Manhattan. In this model, movement pattern of mobile nodes in urban environments is simulated using a grid road topology [10]. This mobility model contains horizontal and vertical roads. Nodes are randomly placed on map at the beginning of simulation and they are allowed to change their lanes. When a node reaches the intersection, it can continue by turning left, turning right, or going straightforward randomly with $0.25,0.25$, and 0.50 probability, respectively.

4.2.3. City Section Model. In this model, movement pattern of mobile nodes in a part of city is simulated [10]. All nodes and streets have particular speed limit in this model. Initial positions of nodes are defined before the simulation beginning. Each node chooses random destination, and a node moves from current location to the destination using shortest travel time between two locations. When the node reaches the destination, node stays there for a specific time and then selects a new random destination by repeating the same process.

4.2.4. Synthetic Model. In this model, movement pattern of mobile nodes is simulated using mathematical equations [19]. In most of the simulations, this model is preferred and the simulation results are compared with real mobility models. This model has some categories, including behavioral models that investigate the movements of driver according to the social interactions, car following model that investigates the car-to-car interaction behavior, stochastic model that creates random motion, and queue model that considers roads as buffer and vehicles on lane as in queue. The most known synthetic models can be summarized as follows.

4.3. Car Following Model. In this model, movement pattern of mobile nodes is simulated according to behavior of each 
driver [20]. CFM is microscopic mobility models. The current speed, length, and current position of cars are used to compute the speed or acceleration of a car. A common equation is used in this model as follows:

$$
X=L+T v_{i}+\mu\left(v_{i}\right)^{2}
$$

where $L$ represents the length of the vehicle, $T$ represents the safe time headway, $v_{i}$ represents the velocity of vehicle, $\mu$ represents the adjusting parameter for deceleration.

4.4. Intelligent Driver's Model. It is a microscopic mobility model and extension of CFM model [21]. IDM is used for freeway and urban traffic as time continuous car following model. Dynamics of the positions and velocities of single vehicles are described in this model. In this mobility model, free acceleration and interaction deceleration are calculated as follows:

$$
\begin{aligned}
& \frac{d v}{d t}=\alpha\left[1-\left(\frac{v}{v_{0}}\right)^{\delta}-\left(\frac{s *(v, \Delta v)}{s}\right)^{2}\right] \\
& s *(v, \Delta v)=s_{0}+v T+\left(\frac{v \Delta v}{2 \sqrt{(\alpha b)}}\right)
\end{aligned}
$$

where $v_{0}$ represents desired velocity, $\Delta v$ represents the velocity difference, $s$ represents minimum spacing, $\alpha$ represents acceleration, $T$ represents the desired time headway, and $b$ represents the comfortable breaking deceleration.

\section{Performance Evaluations}

The simulation studies of IEEE 802.11p and IEEE 802.11b have been performed in NS-2 network simulator [22]. Since Lognormal shadowing path loss model provides more accurate results compared to the other models, that is, Nakagami and Rayleigh models for wireless environments, we have selected it as the channel model [23]. In log-normal shadowing path loss model, the signal to noise ratio $\gamma(d)_{\mathrm{dB}}$ at a distance $d$ from the transmitter is given by

$$
\gamma(d)_{\mathrm{dB}}=P_{t}-\mathrm{PL}\left(d_{0}\right)-10_{\eta} \log _{10}\left(\frac{d}{d_{0}}\right)-X_{\sigma}-P_{\eta}
$$

where $P_{t}$ represents the transmit power in $\mathrm{dBm}, \operatorname{PL}\left(d_{0}\right)$ represents the path loss at a reference distance $d_{0}, \eta$ means the path loss exponent, $X_{\sigma}$ represents a zero mean Gaussian random variable with standard deviation $\sigma$, and $P_{\eta}$ represents the noise power in $\mathrm{dBm}$.

Table 5 summarizes the comparison of the radio propagation models in terms of the assumed propagation condition, line-of-sight (LOS), and communication range. Free space and two-ray ground models assumed that propagation condition is ideal and communication range is circle. On the other hand, due to fading effects, received power is randomly variable in real life. The mean of received power is predicted and reflected in shadowing model. Therefore, the ideal circle is extended to a richer statistic model in shadowing model that gives more realistic results.

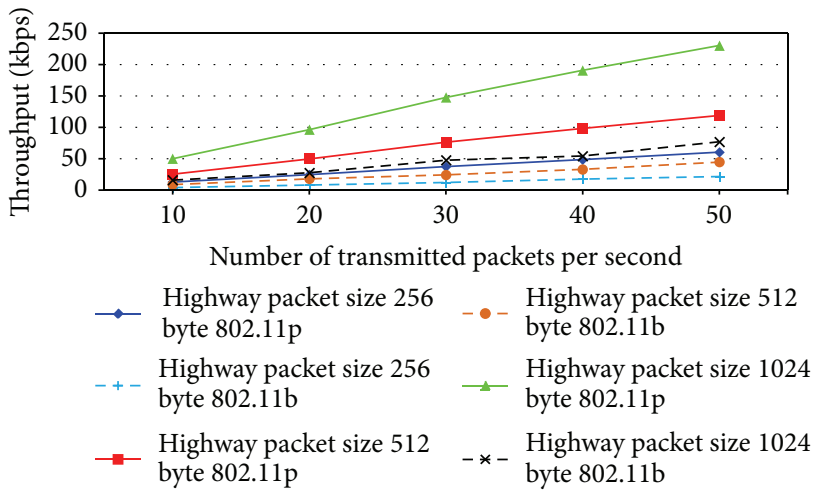

FIGURE 2: Throughput of IEEE 802.11p and IEEE 802.11b in highway environment.

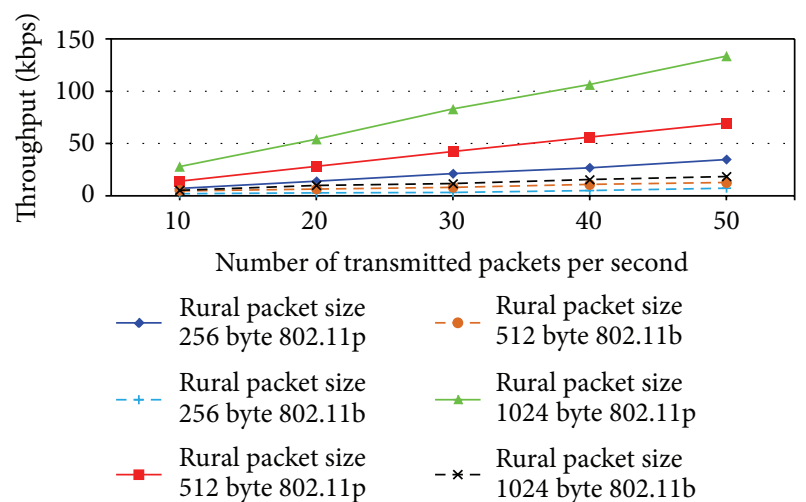

FIGURE 3: Throughput of IEEE 802.11p and IEEE 802.11b in rural environment.

In this study, we have used MOVE traffic simulator tool with NS-2 network simulator and log-normal channel parameters for different traffic environments, that is, highway, rural, and urban areas for performance evaluations. The reason that we used NS-2 and MOVE in our simulations is that both of them are publicly available. Furthermore there are several useful tutorials on web for these simulators. Constant bit rate is selected for traffic type, and different packet sizes are used, including 250, 512, and 1024 bytes with different inter-packet gap (IPG), including 0.1, 0.05, 0.033, 0.025 , and 0.02 second. To statistically analyze the system, we run each simulation 10 times with different seeds and take the average of the measured values. In our simulations, we have used AODV as a routing protocol. The movements of vehicles in simulations are in the same direction with different speed up to average maximum speed. All parameters used in our performance evaluations are listed in Table 6.

The log-normal channel parameters that we used in our performance evaluations are based on the study [12], where parameters have been obtained with set of field tests at $5.9 \mathrm{GHz}$ on highway, rural, and urban environments. The parameters that we used in our simulations for the three environments are given in Table 7 . These environments can be explained as follows.

(i) Highway is an environment that has at least 3 lanes in each direction with no obstacles such as houses, trees, and vehicle speeds are permitted up to $120 \mathrm{~km} / \mathrm{h}$. 


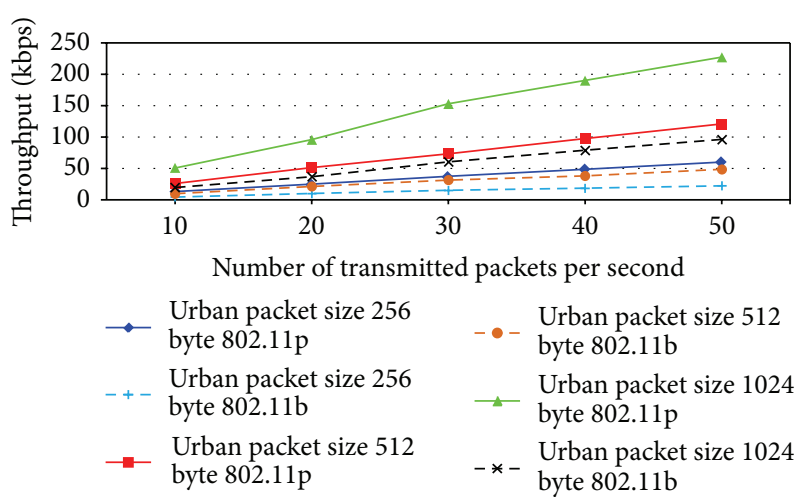

FIGURE 4: Throughput of IEEE 802.11p and IEEE 802.11b in Urban Environment.

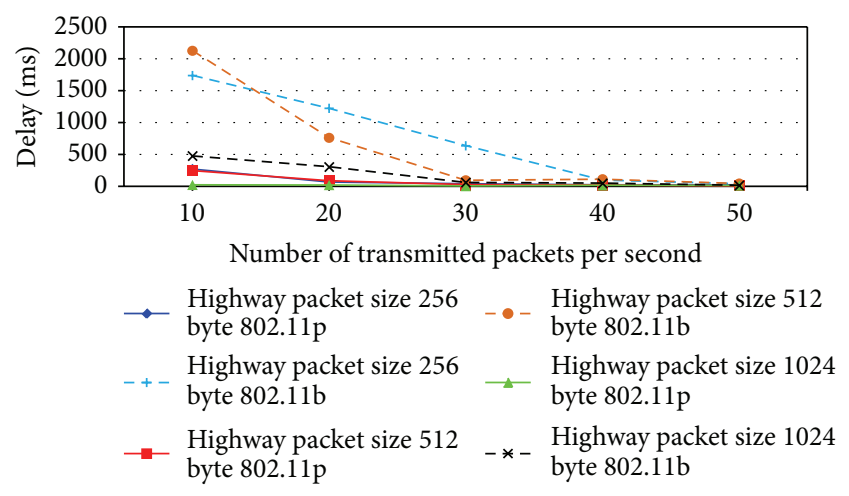

FIgURE 5: End-to-End Delay of IEEE 802.11p and IEEE 802.11b in Highway Environment.

(ii) Rural is an environment that has 1 lane in each direction with trees and hills, and vehicle speeds are permitted between 50 and $90 \mathrm{~km} / \mathrm{h}$.

(iii) Urban is an environment that has 2 lanes in each direction with dense traffic, many traffic lights, junctions and obstacles such as many houses and few trees, and vehicle speeds are permitted between 50 and $70 \mathrm{~km} / \mathrm{h}$.

In these performance evaluations, we have compared the IEEE 802.11p and IEEE 802.11b according to the following performance metrics.

(i) Average Delay means the average time to receive all data on the destination side.

(ii) Delivery Ratio means ratio between the number of successful packets and the total number of transmitted packets.

(iii) Network Throughput means the amount of data transmitted between transceivers in a specific time period.

In our simulations vehicles travel from one point to another point with different speeds up to the maximum speed that is defined previously. There are some traffic lights on ways that vehicles stop at when they returned to red. Furthermore, there are some flows and turn definitions that help the vehicles to reach their final destination. Based on these metrics, we present the performance results from Figure 2 to Figure 12 when different packet size, vehicle speeds, and data rates are employed in different traffic environments. Throughput performance results of IEEE 802.11p and IEEE 802.11b have been shown in Figure 2 to Figure 4. Then, endto-end delay results of IEEE 802.11p and IEEE 802.11b have been shown in Figure 5 to Figure 7 . After that delivery ratio results of IEEE 802.11p and IEEE 802.11 b have been shown in Figure 8 to Figure 10. Finally, the throughput and delivery ratio results of IEEE 802.11p and IEEE 802.11b with different vehicle speeds and different IPG values have been shown in Figure 11 and Figure 12.

Figure 2 shows the network throughput versus number of transmitted packets per second for IEEE 802.11p and IEEE 802.11b in highway environment. When IEEE 802.11p is used as MAC layer protocol, the throughput results are approximately three times better compared to IEEE 802.11b. At the same time, the throughput values are increasing when the number of transmitted packet increases for both IEEE 802.11p and IEEE 802.11b. Since there are no obstacles that affect the $\mathrm{V} 2 \mathrm{~V}$ communication in highway environments, compared to the other environments, that is, rural and urban environment, the throughput results are better in highway environment.

Figure 3 shows the network throughput versus number of transmitted packets per second for IEEE 802.11p and IEEE 802.11b in rural environment. When IEEE 802.11p is used as MAC layer protocol, the throughput results are approximately five times better compared to IEEE 802.11b. There is no big differences on throughput results in IEEE 802.11b protocol. The obstacles, such as trees and hills near the road, affect the $\mathrm{V} 2 \mathrm{~V}$ communication, and this causes lowest throughput ratios compared to the highway and urban environments.

Figure 4 shows the network throughput versus number of transmitted packets per second for IEEE 802.11p and IEEE $802.11 \mathrm{~b}$ in urban environments. The throughput results for IEEE 802.11p are better than IEEE 802.11b. When we increase the packet size in both MAC protocols, throughput results increase significantly. When we compare the throughput results with highway and rural area, it is obviously seen that throughput results of urban area are better than rural area. On the other hand it is worse than highway areas.

Figure 5 shows the end-to-end delay versus number of transmitted packets per second for IEEE 802.11p and IEEE 802.11b in highway environment. When IEEE 802.11p is used as MAC layer protocol, the delay is approximately ten times shorter compared to IEEE $802.11 \mathrm{~b}$. At the same time, the delay between transmitter and receiver is decreasing when the number of transmitted packet increases for both IEEE 802.11p and IEEE 802.11b. For IEEE 802.11p, we got the highest delay for 10 transmitted packets per second when we applied 256 bytes data, and we got the lowest delay when we applied 1024 bytes data.

Figure 6 shows the end-to-end delay versus number of transmitted packets per second for IEEE 802.11p and IEEE 802.11b in rural environment. In rural environment, 
TABLE 5: Comparison of Radio Propagation Models.

\begin{tabular}{lccc}
\hline Propagation model & Assumed propagation condition & LOS & Communication range \\
\hline Free space & Ideal & 1 clear LOS between transmitter and receiver & Circle \\
Two-ray ground & Ideal & 1 Los between 2 mobile nodes & Circle \\
Shadowing & Realistic & Los and Nlos & Extended ideal circle \\
\hline
\end{tabular}

TABle 6: Simulation Parameters.

\begin{tabular}{lc}
\hline Network Simulator & NS-2 \\
Traffic Simulator Tool & MOVE \\
Channel Model & Log-Normal Shadowing \\
Number of Vehicles & 100 \\
Avg. Max. Vehicle Speed & $40-60-80$ \\
Packet Size & $256-512-1024$ Bytes \\
Traffic Type & CBR \\
IPG & 0.1, 0.05, 0.033, 0.025, 0.02 second \\
Queue Type & Drop Tail \\
Traffic Environments & Highway-Rural-Urban \\
Routing Protocol & AODV \\
Vehicle Movements & Same direction with different \\
\end{tabular}

TABLE 7: Log-normal shadowing channel parameters.

\begin{tabular}{lcc}
\hline Environments & Path loss & Shadowing deviation \\
\hline Highway & 1.85 & 3.2 \\
Rural & 1.79 & 3.3 \\
Urban & 1.61 & 3.4 \\
\hline
\end{tabular}

end-to-end delay between transceivers is too much for IEEE 802.11b protocol, and compared to IEEE 802.11p protocol. Like highway environment, delay between transceivers is decreasing when the number of transmitted packet increases for both IEEE 802.11p and IEEE 802.11b. For IEEE 802.11p, the highest delay for 10 transmitted packets per second is calculated when we applied 1024 bytes data for both protocol, and the lowest delay is calculated when we applied 512 and 1024 bytes data for IEEE 802.11p and IEEE 802.11b, respectively.

Figure 7 shows the end-to-end delay versus number of transmitted packets per second for IEEE 802.11p and IEEE 802.11b, in urban environment. In urban environment IEEE 802.11p has lower end-to-end delay compared to IEEE 802.11b. Like highway and rural environment, delay between transceivers is decreasing when the number of transmitted packet increases for both IEEE 802.11p and IEEE 802.11b. When we compare the three environments, urban environment has higher communication delay than both highway and rural environment for two MAC protocols. The highest delay for 10 transmitted packets per second is calculated when we applied 512 and 256 bytes data for IEEE 802.11p and IEEE $802.11 b$, respectively. The lowest delay is calculated when we applied 512 bytes data for IEEE 802.11p and IEEE 802.11b, respectively.

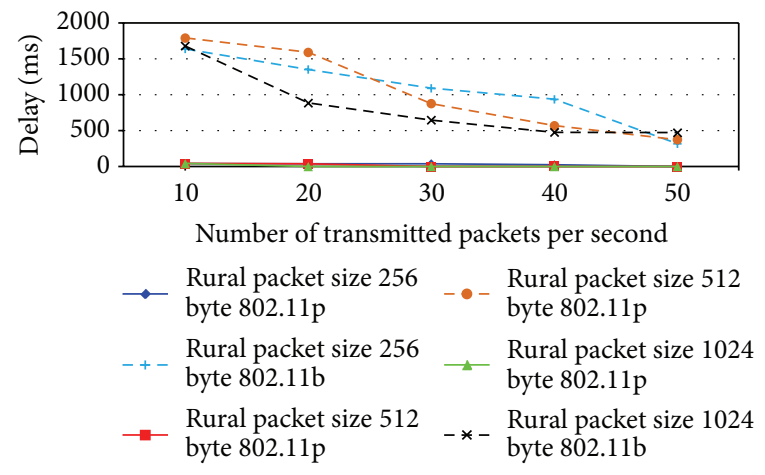

FIgURE 6: End-to-End Delay of IEEE 802.11p and IEEE 802.11b in Rural Environment.

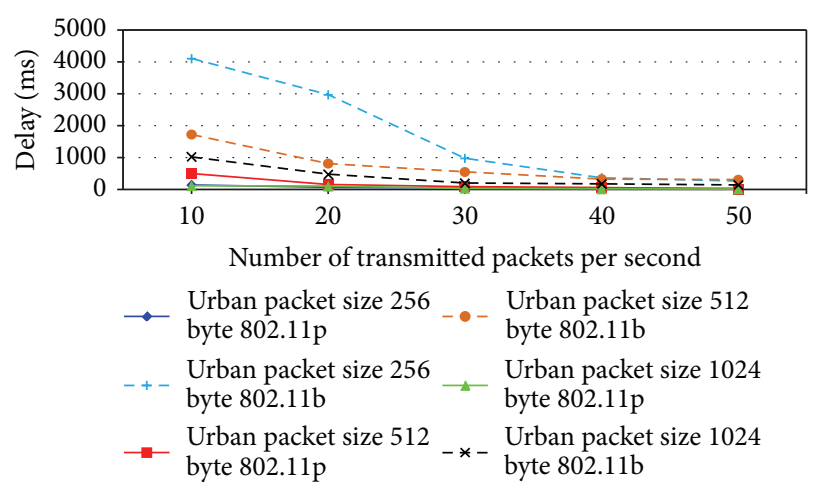

FIGURE 7: End-to-end delay of IEEE 802.11p and IEEE 802.11b in urban environment.

Figure 8 shows the delivery ratio versus number of transmitted packets per second for IEEE 802.11p and IEEE 802.11b in highway environment. When we use IEEE 802.11p as MAC layer protocol, we achieve a delivery ratio between $90 \%$ and $78 \%$. On the other hand delivery ratio is between $31 \%$ and $23 \%$ when IEEE $802.11 \mathrm{~b}$ is used. At the same time, the delivery ratio is decreasing when the number of transmitted packet increases for both IEEE 802.11p and IEEE 802.11b. Compared to the other two environments' delivery ratios, highway has lower delivery ratios. The vehicle speeds and the distance between vehicles may decrease the delivery ratio.

Figure 9 shows the delivery ratio versus number of transmitted packets per second for IEEE 802.11p and IEEE $802.11 \mathrm{~b}$ in rural environment. The delivery ratio of IEEE 802.11 p is between $99 \%$ and $94 \%$, and the delivery ratio of IEEE $802.11 \mathrm{~b}$ is between $34 \%$ and $10 \%$. At the same time, the delivery ratio is decreasing when the number of transmitted packet increases for both IEEE 802.11p and IEEE 


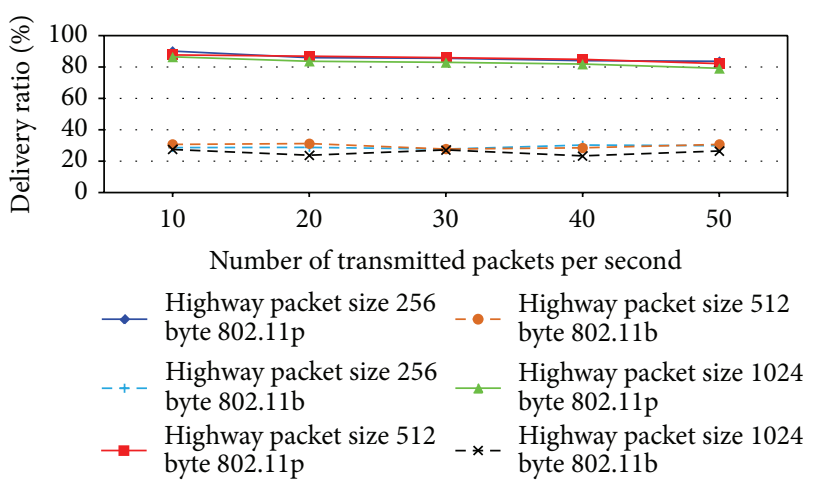

FIGURE 8: Delivery ratio of IEEE 802.11p and IEEE 802.11b in highway environment.

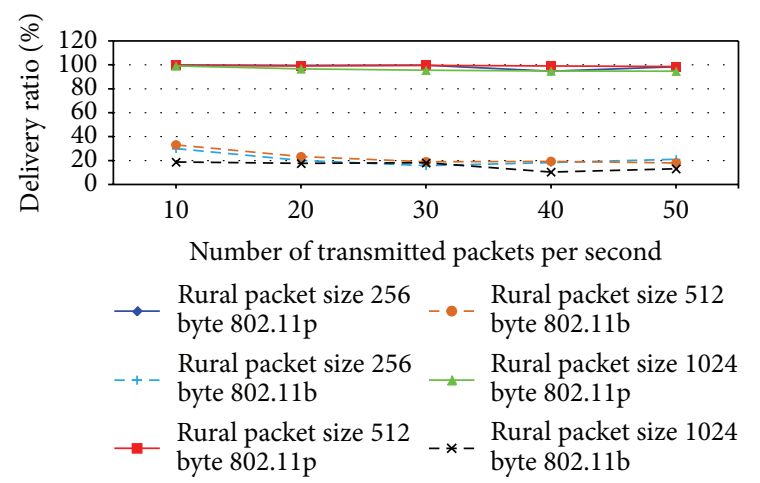

FIGURE 9: Delivery ratio of IEEE $802.11 \mathrm{~b}$ and IEEE $802.11 \mathrm{~b}$ in rural environment.

802.11b. Although there are some obstacles, such as trees and hills, the highest delivery ratios have been obtained in rural environment compared to the other two environments.

Figure 10 shows the delivery ratio versus number of transmitted packets per second for IEEE 802.11p and IEEE 802.11b in urban environment. In urban environment IEEE 802.11p has a delivery ratio between $91 \%$, and $78 \%$ and IEEE 802.11 b has a delivery ratio between $37 \%$ and $30 \%$. At the same time, the delivery ratio is decreasing when the number of transmitted packet increases for both IEEE 802.11p and IEEE 802.11b. Urban environment has higher delivery rate compared to the highway environment. On the other hand, it has lower delivery rate compared to rural environment.

Figure 11 shows the throughput versus packet size for IEEE 802.11p and IEEE 802.11b in urban environment with different vehicle speeds. Although there are no significant throughput differences between two speeds as shown in Figure 11 when we applied $40 \mathrm{~km} / \mathrm{h}$ and $60 \mathrm{~km} / \mathrm{h}$ with $0.1 \mathrm{IPG}$, lower speed vehicles have little higher network throughput. When we apply the different vehicle speeds for IEEE 802.11 , the throughput differences start to increase after applying data packets that have 1024 bytes.

Figure 12 shows the delivery ratio versus packet size for IEEE 802.11p and IEEE 802.11b in urban environment with different vehicle speeds. As shown in Figure 12, vehicles with $40 \mathrm{~km} / \mathrm{h}$ speeds have the highest delivery ratio and vehicles

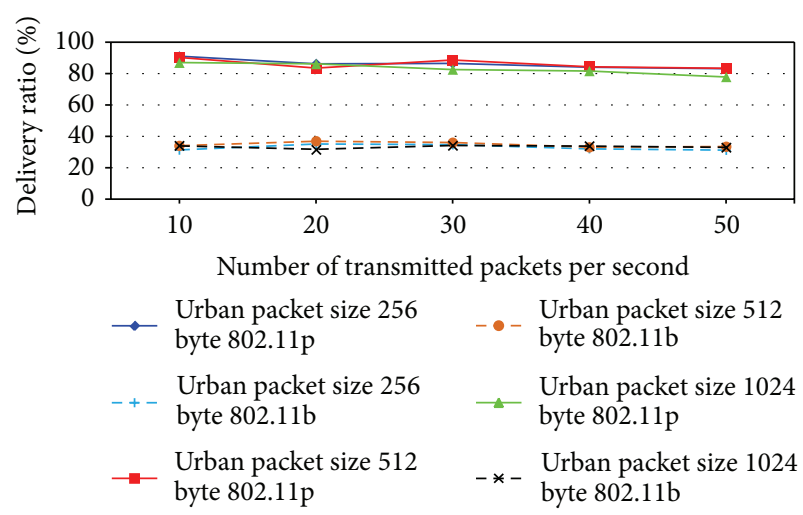

FIGURE 10: Delivery ratio of IEEE 802.11p and IEEE 802.11b in urban environment.

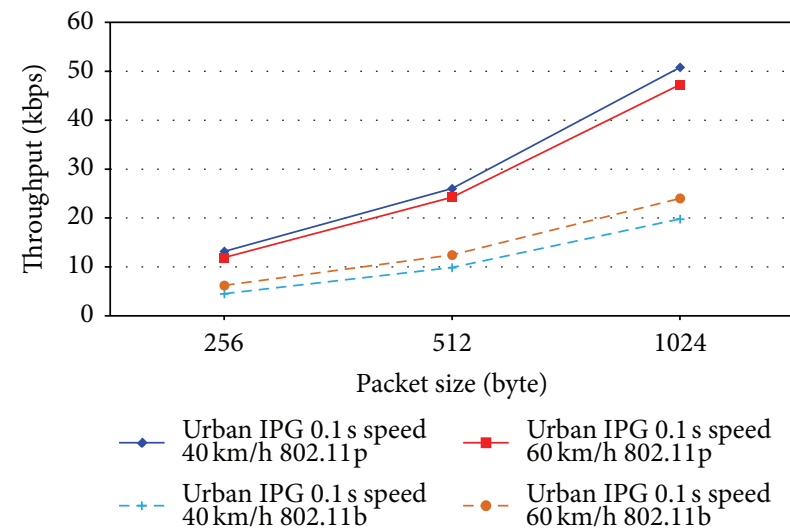

FIGURE 11: Throughput of IEEE 802.11p and IEEE $802.11 \mathrm{~b}$ in urban environment with different vehicle speeds.

with 60 and $80 \mathrm{~km} / \mathrm{h}$ speeds have almost the same delivery ratio for IEEE 802.11p. On the other hand, the delivery ratio of vehicles is increasing when the vehicle speed is increased for IEEE 802.11b. Also, in IEEE 802.11b protocol vehicles with the same speed have almost the same delivery ratio even if the packet size has been increased.

\section{Conclusion}

In this paper, the performance of 802.11p and 802.11b has been compared for different environments, for example, highway, rural, and urban area. We also introduce some simulators that used for VANET simulations and mobility models that are used for these simulators. In our studies, we have used different packet sizes, different interpacket gaps, and different vehicle speeds. Here, our goal is to introduce VANET simulators, research challenges, and mobility models and encourage the research community to explore this promising research area. Overall, comparative performance evaluations show that IEEE 802.11p MAC layer protocol has better results for V2V communications compared to the IEEE 802.11b MAC protocol in terms of network throughput, end-to-end delay, and delivery ratio. Furthermore, the network throughput results of IEEE 802.11p and IEEE 802.11b can be ordered 


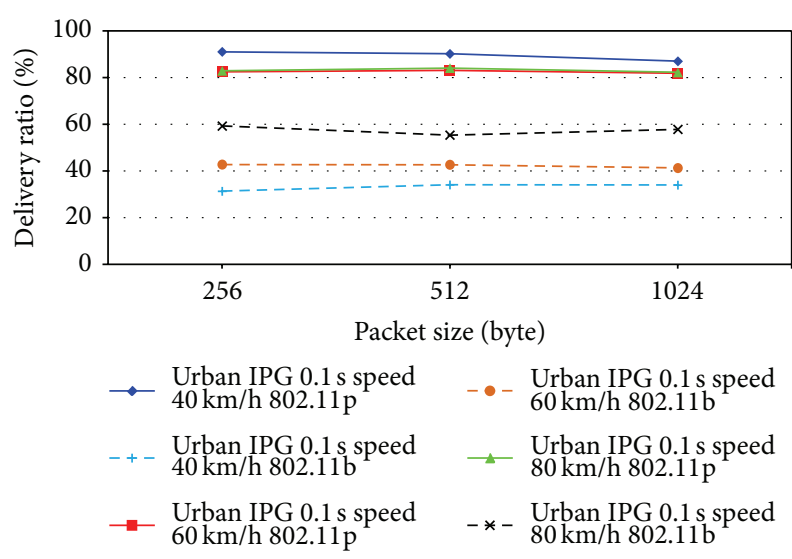

FIGURE 12: Delivery ratio of IEEE 802.11p and IEEE 802.11b in urban environment with different vehicle speeds.

from higher throughput to lower throughput as highway $>$ urban $>$ rural areas. Certainly, there are many important open research issues on $\mathrm{V} 2 \mathrm{~V}$ communications and applications. The development of cross-layer and adaptive protocols for $\mathrm{V} 2 \mathrm{~V}$ communications and applications may be the future work for the researchers.

\section{References}

[1] A. F. Molisch, F. Tufvesson, J. Karedal, and C. F. Mecklenbräuker, "A survey on vehicle-to-vehicle propagation channels," Proceedings of the IEEE Wireless Communications, vol. 16, no. 6, pp. 12-22, 2009.

[2] J. Santa, A. F. Gómez-Skarmeta, and M. Sánchez-Artigas, "Architecture and evaluation of a unified V2V and V2I communication system based on cellular networks," Computer Communications, vol. 31, no. 12, pp. 2850-2861, 2008.

[3] M. Gerla and L. Kleinrock, "Vehicular networks and the future of the mobile internet," Computer Networks, vol. 55, no. 2, pp. 457-469, 2011.

[4] J. Härri, F. Filali, C. Bonnet, and M. Fiore, "VanetMobiSim: generating realistic mobility patterns for VANETs," in Proceedings of the 3rd ACM International Workshop on Vehicular Ad Hoc Networks, pp. 96-97, September 2006.

[5] S. Zeadally, R. Hunt, Y.-S. Chen, A. Irwin, and A. Hassan, "Vehicular ad hoc networks (VANETS): status, results, and challenges," Telecommunication Systems, vol. 50, pp. 217-241, 2010.

[6] T. Willke, P. Tientrakool, and N. Maxemchuk, "A survey of intervehicle communication protocols and their applications," IEEE Communications Surveys and Tutorials, vol. 11, no. 2, pp. 3-20, 2009.

[7] M. Torrent-Moreno, Inter-Vehicle communication: achieving safety in a distributed wireless environment, challenges, systems and protocols [Ph.D. thesis], University Karlsruhe, 2007.

[8] G. Karagiannis, O. Altintas, E. Ekici et al., "Vehicular networking: a survey and tutorial on requirements, architectures, challenges, standards and solutions," IEEE Communications Surveys and Tutorials, vol. 13, no. 4, pp. 584-616, 2011.

[9] G. P. Grau, D. Pusceddu, S. Rea, O. Brickley, M. Koubek, and D. Pesch, "Characterisation of IEEE802.11p radio channel for
Vehicle-2-Vehicle communications using the CVIS platform," Tech. Rep., CAWS internal report, 2010.

[10] G. Grilli, Data dissemination in vehicular networks [Ph.D. thesis], University of Rome, 2010.

[11] H. Hartenstein and K. P. Laberteaux, "A tutorial survey on vehicular ad hoc networks," IEEE Communications Magazine, vol. 46, no. 6, pp. 164-171, 2008.

[12] J. Kunisch and J. Pamp, "Wideband car-to-car radio channel measurements and model at $5.9 \mathrm{GHz}$," in Proceedings of the 68th Semi-Annual IEEE Vehicular Technology (VTC '08), September 2008.

[13] L. Cheng, B. E. Henty, F. Bai, and D. D. Stancil, "Highway and rural propagation channel modeling for vehicle-to-vehicle communications at $5.9 \mathrm{GHz}$," in Proceedings of the IEEE International Symposium on Antennas and Propagation and USNC/URSI National Radio Science Meeting (APSURSI '08), July 2008.

[14] L. Cheng, B. E. Henty, D. D. Stancil, F. Bai, and P. Mudalige, "Mobile vehicle-to-vehicle narrow-band channel measurement and characterization of the $5.9 \mathrm{GHz}$ Dedicated Short Range Communication (DSRC) frequency band," IEEE Journal on Selected Areas in Communications, vol. 25, no. 8, pp. 1501-1516, 2007.

[15] F. K. Karnadi, Z. H. Mo, and K.-C. Lan, "Rapid generation of realistic mobility models for VANET," in Proceedings of the IEEE Wireless Communications and Networking Conference (WCNC '07), pp. 2508-2513, March 2007.

[16] Trans, http://lca.epfl.ch/projects/trans/.

[17] sumo, http://sumo.sourceforge.net/.

[18] F. Bai, N. Sadagopan, and A. Helmy, "Important: a framework to systematically analyze the impact of mobility on performance of routing protocols for adhoc networks," in Proceedings of the IEEE INFOCOM, April 2003.

[19] A. Hassan and T. Larsson, "On the requirements on models and simulator design for integrated vanet simulation," in Proceedings of the International Workshop on Intelligent Transportation System, 2011.

[20] N. P. Vaity and D. V. Thombre, "A survey on vehicular mobility modeling: flow modeling," International Journal of Communication Network Security, vol. 1, no. 4, 2012.

[21] Intelligent Drivers' Model, http://www.wikipedia.org/wiki/Intelligent_driver_model.

[22] ns-2, http://www.isi.edu/nsnam/ns/.

[23] T. Rappapport, Wireless Communications: Principles and Practice, Prentice Hall, 2002. 

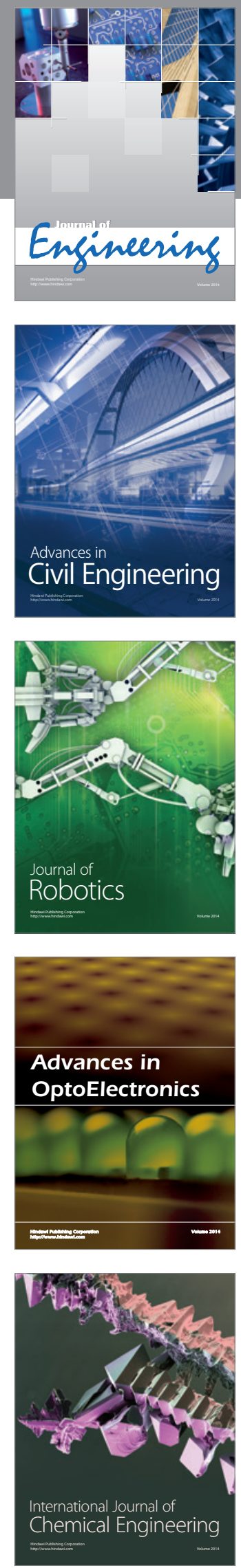

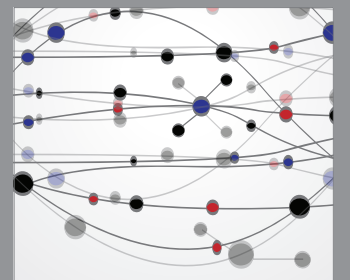

The Scientific World Journal
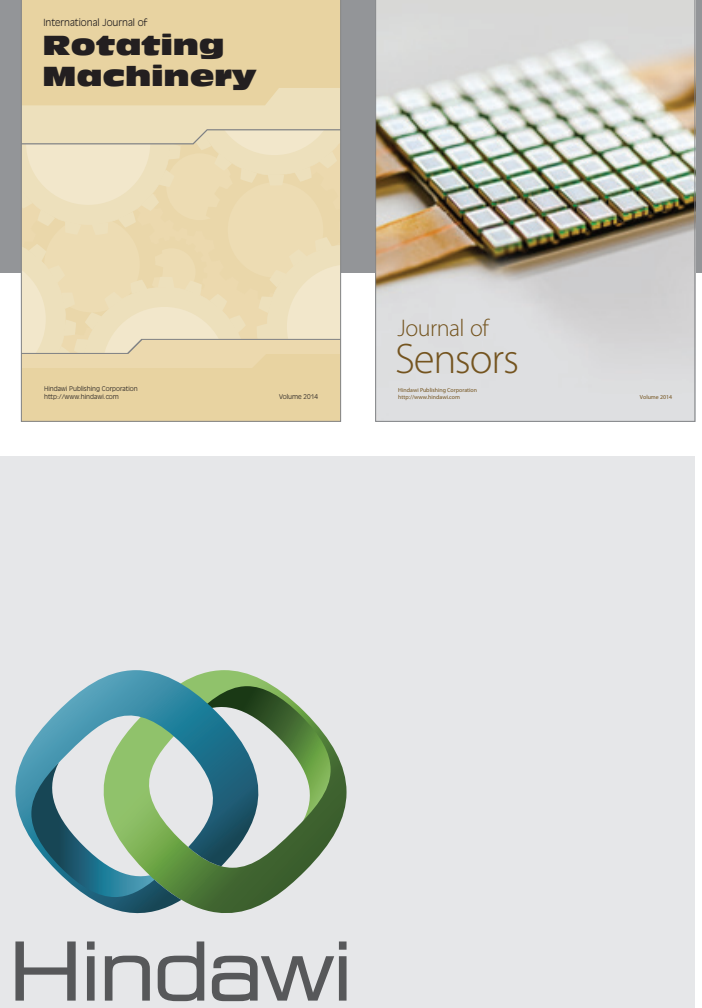

Submit your manuscripts at http://www.hindawi.com
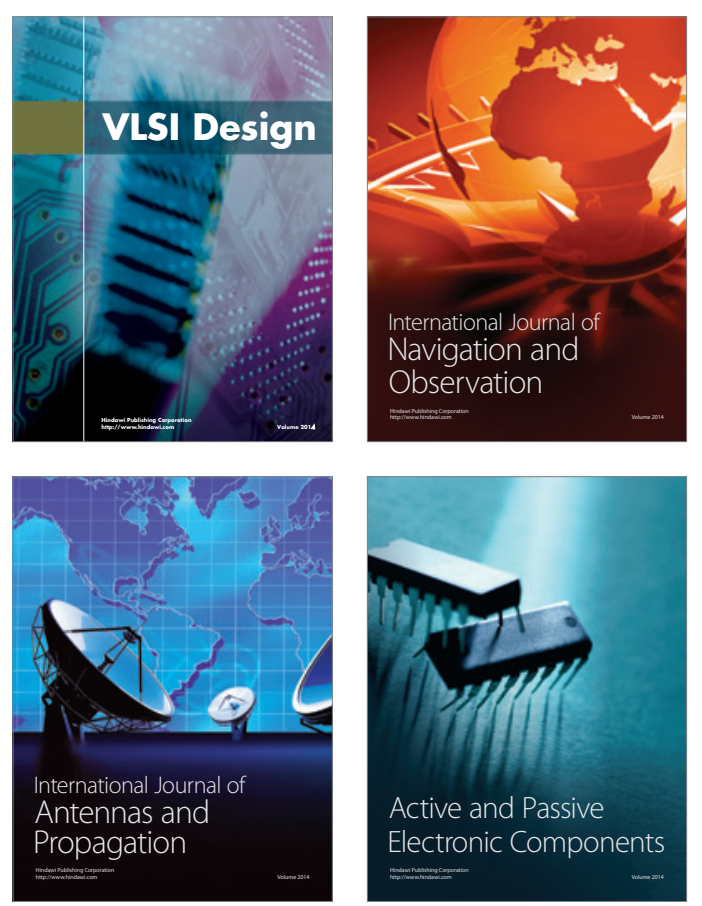
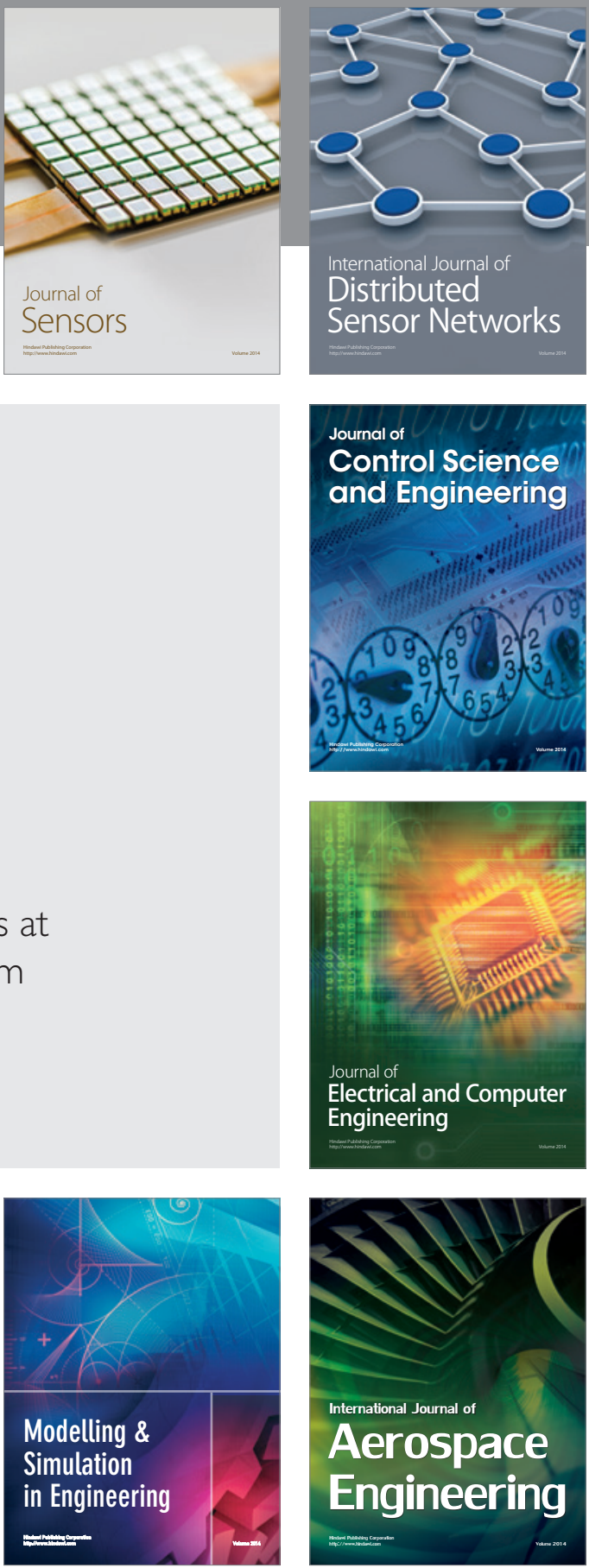

Journal of

Control Science

and Engineering
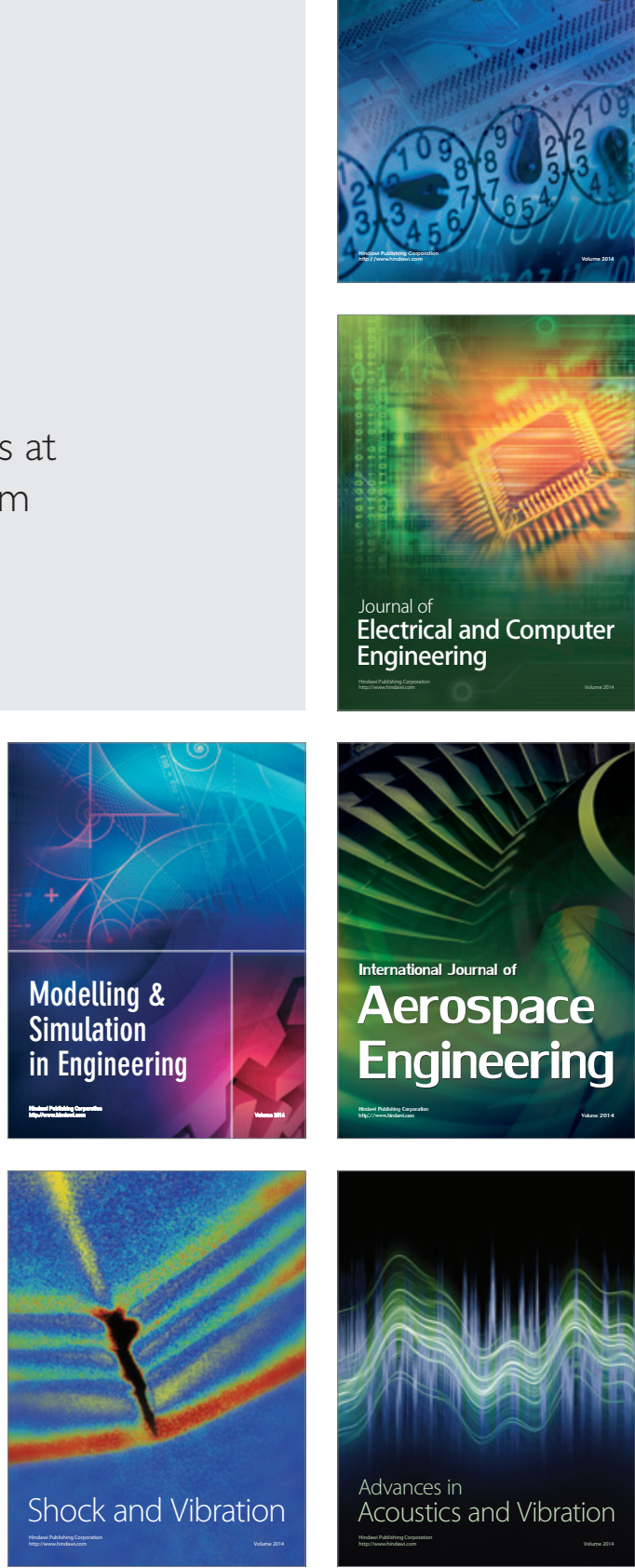